\title{
High-quality conforming hexahedral meshes of patient-specific abdominal aortic aneurysms including their intraluminal thrombi
}

\author{
J. Tarjuelo-Gutierrez $\cdot$ B. Rodriguez-Vila $\cdot$ \\ D. M. Pierce · T. E. Fastl · P. Verbrugghe $\cdot$ I. Fourneau $\cdot$ \\ G. Maleux · P. Herijgers · G. A. Holzapfel · E. J. Gomez
}

\begin{abstract}
In order to perform finite element (FE) analyses of patient-specific abdominal aortic aneurysms, geometries derived from medical images must be meshed with suitable elements. We propose a semi-automatic method for generating conforming hexahedral meshes directly from contours segmented from medical images. Magnetic resonance images are generated using a protocol developed to give the abdominal aorta high contrast against the surrounding soft tissue. These data allow us to distinguish between the different structures of interest. We build novel quadrilateral meshes for each surface of the sectioned geometry and generate conforming hexahedral meshes by combining the quadrilateral meshes. The three-layered morphology of both the arterial wall and thrombus is incorporated using parameters determined from experiments. We demonstrate
\end{abstract}

the quality of our patient-specific meshes using the element Scaled Jacobian. The method efficiently generates high-quality elements suitable for FE analysis, even in the bifurcation region of the aorta into the iliac arteries. For example, hexahedral meshes of up to 125,000 elements are generated in less than $130 \mathrm{~s}$, with $94.8 \%$ of elements well suited for FE analysis. We provide novel input for simulations by independently meshing both the arterial wall and intraluminal thrombus of the aneurysm, and their respective layered morphologies.

Keywords Magnetic resonance imaging - Abdominal aortic aneurysm $\cdot$ Intraluminal thrombus $\cdot$ Conforming hexahedral meshes $\cdot$ Finite element analysis

\section{Introduction}

An abdominal aortic aneurysm (AAA) is a pathological dilatation in a segment of the abdominal aorta, where the aortic diameter is greater than $3 \mathrm{~cm}$ or $50 \%$ greater than the uninvolved proximal vessel [16]. One of the biggest risks associated with this disease is weakening of the aortic wall, which can lead to dissection or rupture of the artery. It is possible that blood stagnates in the dilation, inducing formation of an intraluminal thrombus (ILT) [8]. In such AAAs, three different structures may exist: the AAA wall, ILT and lumen.

A high mortality rate is associated with the rupture of AAAs [19]. Currently, it is not possible to predict when a particular AAA will remain stable or rupture, and treatment by surgical intervention involves significant risks to the patient. Methods currently under investigation to improve rupture risk prediction include those based on numerical simulations, most often using the finite element (FE) method [26]. FE simulations for AAAs can be patient 
specific and permit modeling complex stress states that include effects from the ILT. In the future, such results may improve clinical rupture risk prediction based on preoperative anatomical images.

Recent progress in medical imaging supports clinicians in diagnosis and subsequent treatment for various diseases in different stages. New technologies and methods in magnetic resonance imaging (MRI) as well as computed tomography angiography (CTA) have enhanced soft tissue contrast and enabled clinicians to distinguish between the soft tissue structures of interest. Different acquisition protocols, especially in MRI, open the possibility of differentiating between the AAA wall and the ILT within the context of AAA imaging.

Generating an appropriate computational mesh is prerequisite for applying several numerical techniques, including those based on FE analysis [10]. Such meshes represent the geometry of interest using a set of polyhedral elements. Commonly, these elements are tetrahedra (four connected nodes creating four triangular faces) or hexahedra (eight connected nodes creating six quadrilateral faces). Many fast and robust methods exist in the literature for automatically generating tetrahedral meshes of arbitrary geometries, cf. [7, 14, 29].

Building computational meshes from hexahedra is far more restrictive, and only a limited number of relatively simple geometries can be constructed using high-quality hexahedral elements [23]. However, for a wide range of applications, hexahedral-based meshes are preferred. First, to achieve the same solution, accuracy for a given FE analysis requires far more tetrahedral elements then hexahedral elements, and this leads to higher computational costs (both time and memory) [6, 21]. Even more, when the aim is to apply FE analysis, tetrahedral meshes produce acceptable displacement results but are relatively inaccurate for predicting stresses $[18,20]$. Hexahedral mesh generation is constrained by the need to decompose a desired geometry (repeatedly) into simpler sub-geometries that can be meshed automatically $[11,17]$.

In the specific case of vascular structures, generating hexahedral meshes is especially challenging due to the complex 3D branching anatomy. Hence, we propose a specific algorithm designed to work with patient-specific geometries obtained from preoperative imaging. Each patient's aneurysm is unique, characterized by its location and shape, and must be accurately represented for subsequent analyses to be meaningful. About $90 \%$ of abdominal aneurysms are located below the renal arteries [16]. The histological structure and the mechanical characteristics of the infra-renal aorta differ from those of the thoracic aorta [28]. Thus, our investigation focuses on modeling the lower part of the aorta and the aortic bifurcation into the iliac arteries.

The geometry under study is roughly described as a tubular structure in the shape of an inverted Y, distorted by the aneurysm and ILT wherever present. Regarding shape, an aneurysm can be fusiform or saccular. Fusiform aneurysms are most common and bulge the whole perimeter of the aorta, while saccular aneurysms bulge only on one side. We account for both cases with our method. If blood stagnates in the aneurysm and forms an ILT, it exists as a conforming structure with the aortic wall, i.e., the ILT's outer contour is the same as the wall's inner contour. Therefore, resulting meshes must also be conforming.

A fully automatic algorithm for generating hexahedral meshes of arbitrary geometries has not yet been achieved. Furthermore, to the authors' knowledge, there are no automatic algorithms which provide automatic meshing of bifurcating structures. The literature on this topic generally deals with planar bifurcations, i.e., centerlines which evolve in a single plane, e.g., [15, 27]. Antiga et al. [1] proposed a method for generating high-quality hexahedral meshes of bifurcating vessels using the Cooper scheme, based on reconstructing 3D models with a level set technique, analyzing them with a skeletonization algorithm and automatically decomposing the result into branches. Later, Antiga and Steinman [2] developed a decomposition and mapping method based on centerlines which consists of robustly decomposing the surface into its constituent branches and mapping each branch onto a template parametric plane.

De Santis et al. [3-6] proposed several methods based on combinations of surface slicing, spline reconstruction and mapping. For example, these authors developed methods to generate conforming, structured hexahedral meshes from triangulated surfaces [5] or from centerline-based synthetic descriptors (centerlines, radii and centerline normals) in combination with block structures [6]. In this latter work [6], the authors presented conforming meshes of the arterial wall and its lumen, but they have not segmented the outer contour of the wall from the surrounding soft tissues, and the wall is thus reconstructed from the lumen contour by assuming a constant or radius-dependent wall thickness. Furthermore, they did not account for the ILT.

In this study, we present a novel and robust method for generating conforming hexahedral meshes of both the aortic wall and the ILT. We construct these meshes using largely high-quality elements, especially at the bifurcation, that are suitable for FE analysis of tissue stresses. Our method accounts for the evolution of the vessel's centerline, which may develop outside a single plane, and generates the mesh directly from segmented images without the requirement to reconstruct triangular surfaces.

\section{Methods}

We detail our method for generating two conforming (largely) hexahedral meshes, one for the AAA wall and 
another one for the ILT, based on patient-specific MRI data. To achieve this goal, we start from MRI data which we generate using a protocol developed to give the abdominal aorta high tissue contrast against the surrounding soft tissue. The quality of these data allows us to segment contours representing the ILT where present, as well as the lumen and outer arterial edge. We then construct three quadrilateral meshes: one represents the outer surface of the arterial wall; another representing the inner arterial wall; and a third representing the inner surface formed by the thrombus, but complemented by the lumen surface where the thrombus is not present.

If one seeks to generate regularly shaped hexahedral elements, the irregular geometry of the bifurcation presents a serious difficulty, and hence, our main challenge in this work is generating high-quality elements at the aortic bifurcation into the iliac arteries. We detail a novel method to handle meshing of the bifurcation region. We build a series of quadrilateral meshes based on partitioning this region. Finally, we combine the quadrilateral meshes to form conforming hexahedral meshes. We demonstrate the performance of our method on four MRI studies of patients with AAAs near the aortic bifurcation into the iliac arteries.

We implemented our method using MATLAB R2009a (Mathworks Inc., Natick, MA, USA).

\subsection{Image data and segmentation}

Different MRI acquisition protocols open up the possibility of differentiating between different soft tissue structures. We developed an MRI protocol focused to provide high contrast for the AAA wall and the ILT against the surrounding soft tissue. If no contraindications existed, we perform MRI studies using a $1.5 \mathrm{~T}$ Aera scanner (Siemens, Erlangen, Germany) on patients with AAAs of diameter larger than $5 \mathrm{~cm}$. We registered the MRI study at the clinical trial center of the University Hospital of Leuven (study number S52774) and obtained ethical approval from the ethical committee UZ Leuven.

Regarding the MRI data specifically, we use a sagittal and transversal balanced steady-state free precession (bSSFP) sequence as a localizer, with 20 sagittal slices of $5 \mathrm{~mm}$ slice thickness and 30 transversal slices of $6 \mathrm{~mm}$ slice thickness, both with no intersection gap, a field of view (FOV) of $380 \mathrm{~mm}$, a matrix size of $320 \times 260$, a time to repetition/time to echo (TR/TE) of $4.41 \mathrm{~ms} / 2.21 \mathrm{~ms}$, a flip angle of $62^{\circ}$ and a one signal average. Thereafter, we completed a pulse triggered, three slice T1 Turbo Spin Echo (TSE) sequence with $6 \mathrm{~mm}$ slice thickness, TR/TE of $800 \mathrm{~ms} / 62 \mathrm{~ms}$, FOV of $160 \mathrm{~mm}$ and a matrix of $256 \times 256$ and a flip angle of $180^{\circ}$. Next, we perform a coronal breath-hold fast low-angle shot (FLASH) 3D sequence after intravenous administration of a standardized dose of
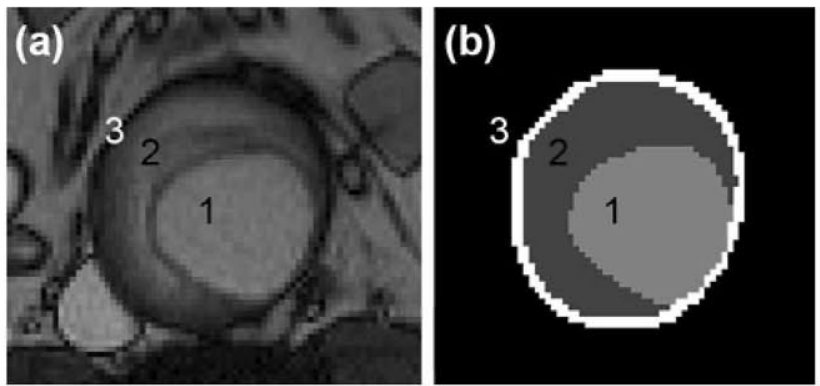

Fig. 1 Images of a representative abdominal aortic aneurysm where the three structures of interest are observed ( 1 lumen, 2 intraluminal thrombus and 3 aortic wall): a magnetic resonance image; b segmented image containing contours of the structures

$0.1 \mathrm{mmol} / \mathrm{kg}$ Gd-DOTA (Dotarem, Guerbet, France). We execute this in the arterial phase with a slab thickness of 96 slices of $1.25 \mathrm{~mm}, 384 \times 336$ matrix size, TR/TE of $3.04 \mathrm{~ms} / 1.09 \mathrm{~ms}$ and a FOV of $400 \mathrm{~mm}$ and a flip angle of $25^{\circ}$.

After acquisition, we evaluate image quality and tissue contrast for the AAA wall and the ILT in order to select images which best meet our objectives. After analysis, we select the transversal bSSFP sequence for segmentation and reconstruction of the patient-specific geometry (see Fig. 1a). We use three 3D binary images of the aortic wall, the ILT and the lumen for each patient. For each structure, we extract contours from the binary images (see Fig. 1b). After segmentation, we apply a Gaussian smoothing to remove inaccuracies (unlikely edges due to noise or manual segmentation errors), where we take care to avoid distorting the shape of the structures of interest, e.g., reducing the contours to circles or ellipses. Although bare contours may be applied, linear interpolation is used between the points obtained from the manual segmentation performing a smoothing process results in softer and more realistic representations. The smoothing process applies a Gaussian filter $(\sigma=3)$ to the contours obtained from the segmentation, starting at the first point of the contour. We repeat this process starting at the last point of the contour such that the process is independent of position and to avoid distortion. The final result is an average of these smoothed contours. The process thus removes only high frequency features.

\subsection{Bifurcation modeling}

\subsubsection{Division of the bifurcation into vessels}

Our method for modeling the bifurcation begins by dividing it into three distinct vessels, following Lee et al. [13]. We perform this division by defining three planes, which intersect in a single straight line. Figure 2 illustrates the three planes in a representative patient-specific mesh: 


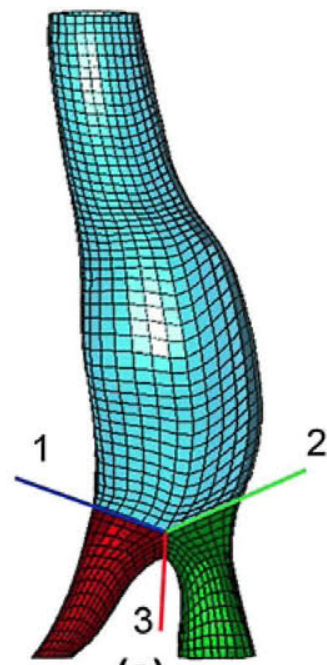

(a)

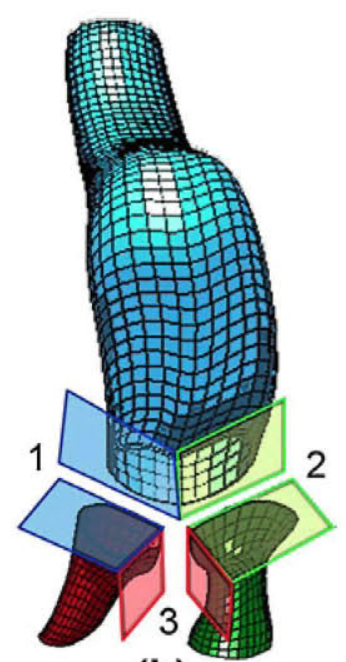

(b)
Fig. 2 Three planes at the bifurcation, used to develop a representative patient-specific mesh: a plane and vessel position at the bifurcation; $\mathbf{b}$ expanded view with planes and isolated vessels



Fig. 3 A representative set of patient-specific contours showing the position of the points required to divide the bifurcation region into distinct vessels (with anterior $(A)$; posterior $(P)$; bottom $(B)$; left $(L)$; right $(R)$ ) and the arcs defined by the intersection of the resulting planes and the contours

planes 1, 2 and 3 in Fig. 2a and expanded in Fig. 2b. We define each plane by the respective common line and an additional point. The positions of these three points, and the two points needed to define the original straight line, are critical as these determine the quality of the division and the resulting mesh. We place these five points as follows: anterior $(A)$ - on the lumen surface, in front of and above the bifurcation; posterior $(P)$ - on the lumen surface, in back of and above the bifurcation; bottom $(B)$-on the wall surface, in the bottom part of the bifurcation; left $(L)-$ on the lumen surface and above the anterior $\mathrm{A}$ and posterior $\mathrm{P}$ points; right $(R)$-on the lumen surface and above the points $A$ and $P$. Points $A$ and $P$ define the common straight line and must be located above the iliac arteries. Point $B$ must be placed below the aorta, and points $L$ and $R$ must be located in a position above the line $A P$. Figure 3 illustrates the position of these five points on the bifurcation of a representative set of patient-specific contours.

\subsubsection{Division of the vessels into sections}

Next, we calculate the intersections of these three planes with the segmented contours (arcs in the example shown in Fig. 3). Points $A$ and $P$ define the direction (we define a unit vector $\overline{\mathrm{AP}}$ ) used for dividing the three vessels into sections (we divide the vessel of the abdominal aorta into a left and a right section, while we divide the iliac arteries into interior and exterior sections). Thereby, we divide the volume into six sections, which we model independently. Using this method, we control the number and quality of the quadrilaterals generated in each section using six parameters, which we adjust to tune the mesh density and quality. We define these six parameters as the longitudinal divisions of the aorta $\left(L_{\text {Aorta }}\right)$ and the iliac arteries $\left(L_{\text {RIliac }}\right.$, $\left.L_{\mathrm{LIliac}}\right)$, and circumferential divisions of the right $\left(C_{\text {Right }}\right)$, left $\left(C_{\text {Left }}\right)$ and bottom $\left(C_{\text {Bottom }}\right)$ sides.

We require care to achieve meshes at the transition zones between all six independent sections sufficient for subsequent use in FE simulations. In our process for defining these regions, we pay special attention to transition zones between the different sections to avoid poor mesh quality. For each closed vessel contour, we calculate the lumen centerline using the centroid of the enclosed area ( $C$ in Fig. 4a, and $C_{1}$ and $C_{2}$ in Fig. 4b). The composition of these centroid points determines the lumen centerline. Next, we apply a separate process for the segments of the aorta and those of the iliac arteries. For the aorta contours, we apply the vector $\overline{\mathrm{AP}}$ (anterior-posterior) at the center of each contour (see Fig. 4a). We extend this vector in both directions to obtain the two intersection points of this extended vector and the vessel contour. We assign each contour to its corresponding region (left or right) and set the number of divisions in each region according to the aforementioned parameters $C_{\mathrm{Right}}$ and $C_{\mathrm{Left}}$.

We modify our process for defining these regions for axial slices with two contours (i.e., in the iliac arteries) since we intend that the size of the external regions is greater than the internal ones (see Fig. 4b). Again, we apply the vector $\overline{\mathrm{AP}}$ at the center of each contour and determine the intersections of their extensions with the two vessel contours. However, we need two additional points to define the required regions. We obtain these additional points in the following. First, we determine the point $M$ at the midpoint of the line segment from point $A$ to point $P$ (originally used to determine the vector $\overline{\mathrm{AP}}$ ). The projection of $M$ normal to the imaging planes onto the current imaging 


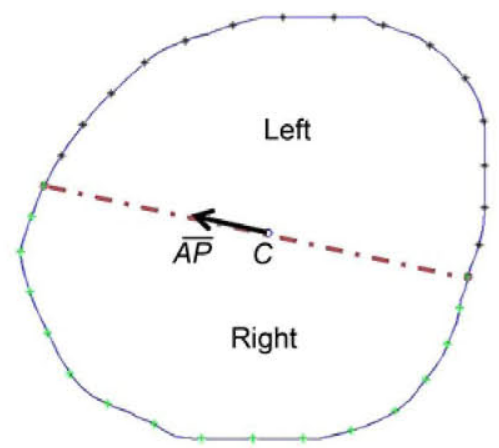

(a)

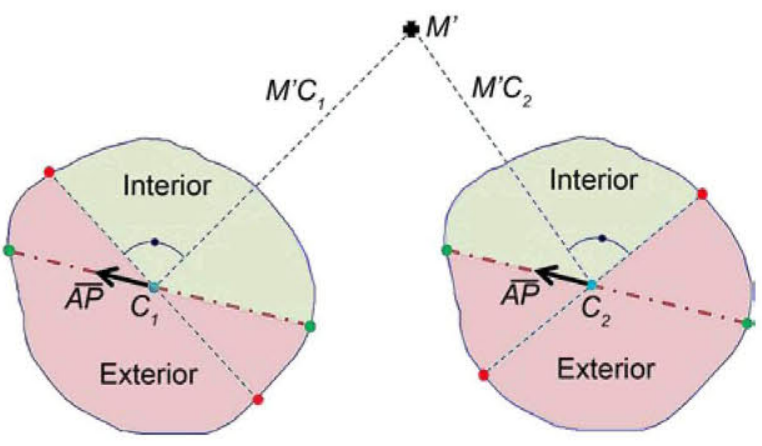

(b)

Fig. 4 Representative arterial contours from two different imaging planes: a aortic artery with area center $C$, vector $\overline{\mathrm{AP}}$ and resulting regions for meshing; $\mathbf{b}$ iliac arteries with projection point $M^{\prime}$, area centers $C_{1}$ and $C_{2}$, vectors $\overline{\mathrm{AP}}$ and resulting regions for meshing

Fig. 5 Representative imagebased contours used for quadrilateral mesh and centerline generation: a initial axial contours; b longitudinal lines; $\mathbf{c}$ resulting quadrilateral mesh; $\mathbf{d}$ resulting set of center points

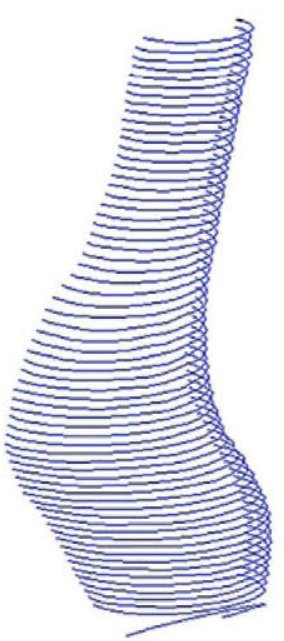

(a)

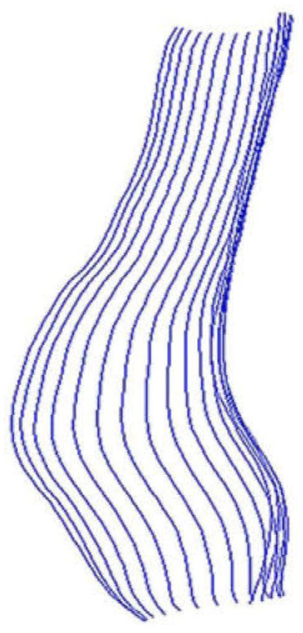

(b)

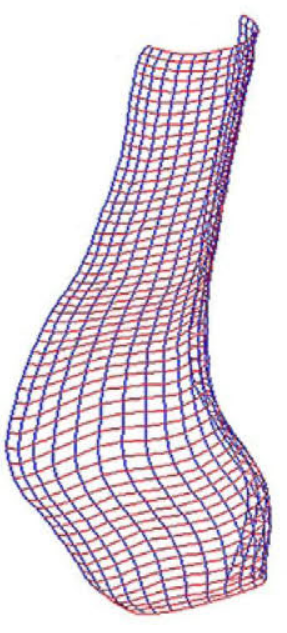

(c)

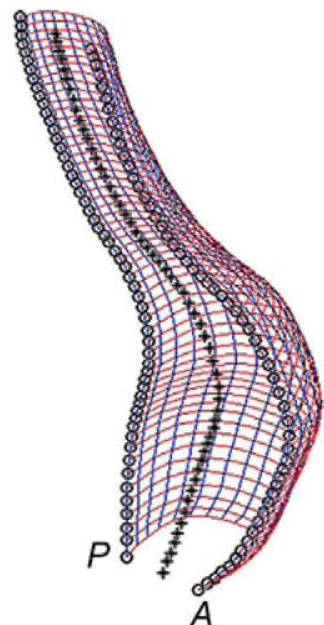

(d) plane with the two vessel contours determines the location of $M^{\prime}$. Next, we link the point $M^{\prime}$ with the centers of the iliac contours $C_{1}$ and $C_{2}$ to determine the line segments $M^{\prime} C_{1}$ and $M^{\prime} C_{2}$. For each of these segments, we calculate a perpendicular segment through the center of each vessel contour. The intersections of these segments and the vessel contours provide the second set of desired points. Thus, we calculate four intersection points on each vessel contour and define the internal region using two points that form the smallest segment of the vessel contour that intersects the segment $M^{\prime} C_{1}$ in the case of the first vessel contour (or $M^{\prime} C_{2}$ in the case of the second vessel contour).

\subsection{Quadrilateral mesh and centerline generation}

Once we determine the different sections and set the corresponding parameters, we build a quadrilateral mesh of each section. We divide the initial axial contours of the regions (see Fig. 5a) according to the circumferential division parameters $C_{\mathrm{Right}}, C_{\mathrm{Left}}$ and $C_{\mathrm{Bottom}}$, with the points equally spaced over the contours. The union of corresponding points from each axial contour generates a set of longitudinal lines (see Fig. 5b). We divide these lines according to the longitudinal division parameters $L_{\text {Aorta }}, L_{\text {RIliac }}$ and $L_{\mathrm{LIliac}}$. The resulting points are not equally spaced along the longitudinal lines due to the dilation of the AAA. We space the points further apart the closer they are to the bifurcation. Once again, the union of corresponding points results in a new set of circumferential lines. We combine the sets of circumferential and longitudinal lines to form a mesh of quadrilateral elements (see Fig. 5c). We apply this process for the axial contours generated from the lumen surface, ILT and outer edge of the arterial wall.

In order to build hexahedral meshes from resulting quadrilateral meshes, we require a set of points along the lumen centerline for each axial contour parallel to the imaging planes. We calculate these points from our quadrilateral mesh of the lumen surface. We follow the longitudinal 
line containing point $A$ and the longitudinal line containing point $P$, and for each axial contour, we determine midpoint of the line segment between the corresponding points. We use these midpoints as the lumen centerline data (see Fig. 5d).

\subsection{Hexahedral mesh generation}

In order to generate two conforming hexahedral meshes, one of the aortic wall and one of the ILT, we require the lumen center points and the three quadrilateral meshes, where we take one of them as a reference. The choice of the reference mesh is arbitrary, and in this case, we have chosen the quadrilateral mesh of the external surface of the arterial wall. To ensure generation of undistorted hexahedral elements, we first refine the quadrilateral elements of the remaining meshes (the inner arterial wall and ILT surface meshes). We perform a ray-casting technique based on the reference mesh. Thereby, we trace a ray from each point of the mesh to its associated center point. Wherever this ray intersects with the surface defined by the quadrilateral mesh of the inner face of the arterial wall, we define a new point. We use this new set of points to update the original quadrilaterals and thereby refine the mesh of the internal face. Finally, we construct the desired hexahedral mesh of the arterial wall by joining the quadrilaterals of the external and internal faces of the wall.

Similarly, we repeat this process for generation of a hexahedral mesh of the ILT. In this case, our reference is the set of points determining the new quadrilateral mesh of the inner face of the arterial wall. Again, we apply a ray-casting technique with the lumen centerline points to construct a new quadrilateral mesh for the inner surface of the ILT (or repeating the lumen surface where ILT is not present). We then join the quadrilateral meshes of both the inner face of the ILT and the inner face of the arterial wall to build a hexahedral mesh of the ILT. We remove any hexahedral elements with zero volume, i.e., wherever the ILT does not exist, so that the inner face of the ILT is equal to the lumen surface. Since the ILT is not necessarily present along the whole AAA, the interface between surfaces with ILT and without ILT will generate coincident nodal points. Along this interface, at the edges of the ILT, we define collapsed hexahedral elements (generalized elements with less than eight distinct nodes) by merging coincident nodes, in order to obtain softer transitions, cf. [30].

Both the arteries and thrombi constitute distinct layered morphologies, and each of these layers has a distinct histological structure and different mechanical properties. In order to generate patient-specific meshes which account for the layered morphology of both the arteries (intima, media and adventitia) and thrombi (luminal, medial and abluminal portions), we use experimentally determined through the thickness ratios. We partition elements of the aortic wall into three elements along the radial direction, representing intima, media and adventitia. In the same way, we partition elements of the thrombus mesh into three layers representing the luminal, medial and abluminal thrombi layers [25]. Ratios for the arterial layers, relative to the composite arterial wall, are 0.19 for the intima, 0.44 for the media and 0.37 for the adventitia, cf. [22]. In the thrombus, the layer ratios are 0.38 for the luminal, 0.38 for the medial and 0.24 for the abluminal layer [25]. With this partitioning process, we alter neither the longitudinal nor circumferential topology of the meshes, and hence, the originally conforming structures retain this property.

\subsection{Inspection of mesh quality}

Within the context of the FE method, the quality of the finite elements obtained from the mesh generation greatly affects both the convergence of the simulations and the resulting approximations to the solutions of the governing partial differential equations. Additionally, accuracy in the representation of the true, patient-specific in vivo geometry also influences the applicability of the results [12]. Metrics for mesh quality must detect inverted elements (elements with negative volume, which generate meaningless results or prevent solution convergence) and provide an estimate of the mesh's fitness for use in numerical simulations.

Several factors affect the quality of a FE mesh, i.e., its usefulness in numerical simulations, and these depend on the type of calculation and on the desired results. For the analysis of solid structures, the Scaled Jacobian is a common quality metric [6]. We evaluate the Jacobian $J_{k}$ at the element center $k=0$ using the principal axes, and at each node $k, k \in[1, \ldots, 8]$, of each hexahedral element as the triple scalar product of the edges connected to that node $\left(\boldsymbol{e}_{k 1}, \boldsymbol{e}_{k 2}, \boldsymbol{e}_{k 3}\right)$ using

$J_{k}=\boldsymbol{e}_{k 1} \cdot\left(\boldsymbol{e}_{k 2} \times \boldsymbol{e}_{k 3}\right)$.

The modulus of (1) represents six times the volume of the tetrahedron enclosed by these three edges. We evaluate the Scaled Jacobian of an element as the minimum of each nodal Jacobian $\left(J_{k}\right.$ from (1)) divided by the length of the three corresponding edges $\left(\boldsymbol{e}_{k 1}, \boldsymbol{e}_{k 2}, \boldsymbol{e}_{k 3}\right)$ using

Scaled Jacobian $=\min _{k \in[0, \ldots, 8]}\left[\frac{J_{k}}{\left\|\boldsymbol{e}_{k 1}\right\|\left\|\boldsymbol{e}_{k 2}\right\|\left\|\boldsymbol{e}_{k 3}\right\|}\right]$,

where the Scaled Jacobian takes the range $[-1,1]$ for a hexahedral element, with -1 corresponding to the worst possible elements and +1 the best possible ones (NB: only elements with a nonzero, positive Scaled Jacobian are suitable for FE analysis [12]). The Scaled Jacobian applies only to regular hexahedral elements having eight (distinct) vertices at different spatial locations in 3D space. Hence, 


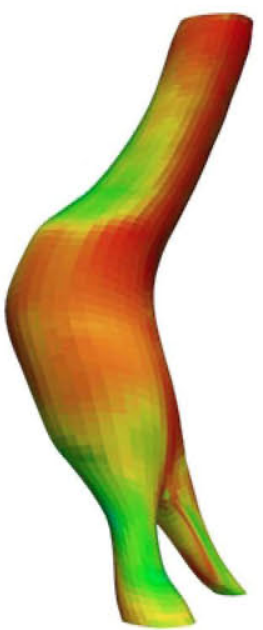

(a)



(e)

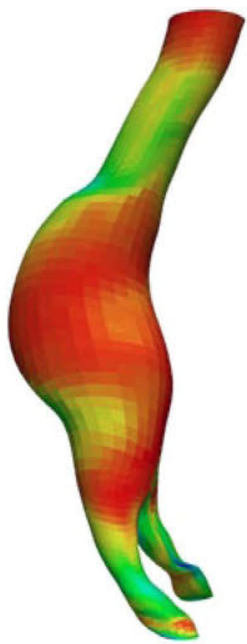

(b)

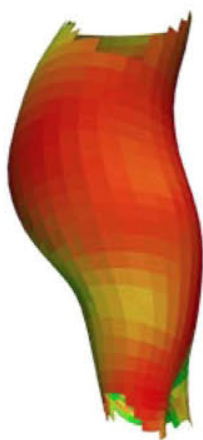

(f)

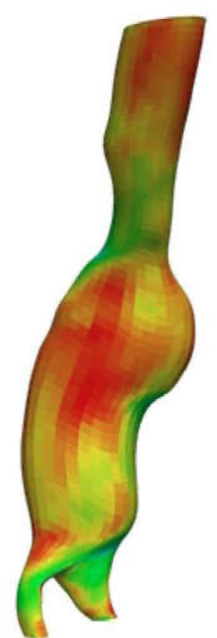

(c)



(g)



(d)



(h)
Scaled Jacobian [-]

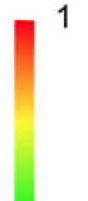

0.5
Fig. 6 Resulting hexahedral meshes for four representative abdominal aortic aneurysms (AAAs) and their corresponding intraluminal thrombi (ILTs) using the Scaled Jacobian as a measure of element

we remove elements with collapsed nodes from our subsequent figures and statistics. We use the open source program Paraview (Kitware, Inc., Clifton Park, New York, USA) to evaluate the Scaled Jacobian, among other quality metrics, which in turn uses the Verdict library [9, 24].

\section{Results}

We test our method on four representative MRI studies of patients with AAAs near the aortic bifurcation into the iliac arteries. Once we select our five bifurcation points manually $(A, P, B, L$ and $R$ ), we are able to generate conforming hexahedral meshes, e.g., containing 125,000 elements in approximately $130 \mathrm{~s}$ using an Intel Core2Duo processor E8400 at three GHz, eight GB RAM and a 64-bit operating system. quality: a-d AAA walls; e-h corresponding ILTs (NB: we do not include collapsed elements in this analysis)

The individual thrombi have distinctly different shapes (with fusiform or saccular aneurysms) and are located at different positions along the aorta and within the AAA. In all cases, our method generates high-quality elements modeling the arterial wall (including particularly the bifurcation) and ILT, as shown in Fig. 6. Figure 6a-d illustrates the resulting conforming hexahedral meshes of the arterial wall, and Fig. 6e-h illustrates their corresponding ILTs (not to scale). We shade the meshes in Fig. 6a-h according to the Scaled Jacobian of the mesh elements, recall that we exclude elements with collapsed nodes from our figures.

Figure $7 \mathrm{a}$ illustrates, for the four representative MRI studies, the distribution of the Scaled Jacobian values for the separated layers of the AAA walls, while Fig. 7b illustrates the same for the separated layers of the ILTs. In both figures, bars represent the arithmetic means, while bracketed lines represent the standard errors. 
(a) AAA wall

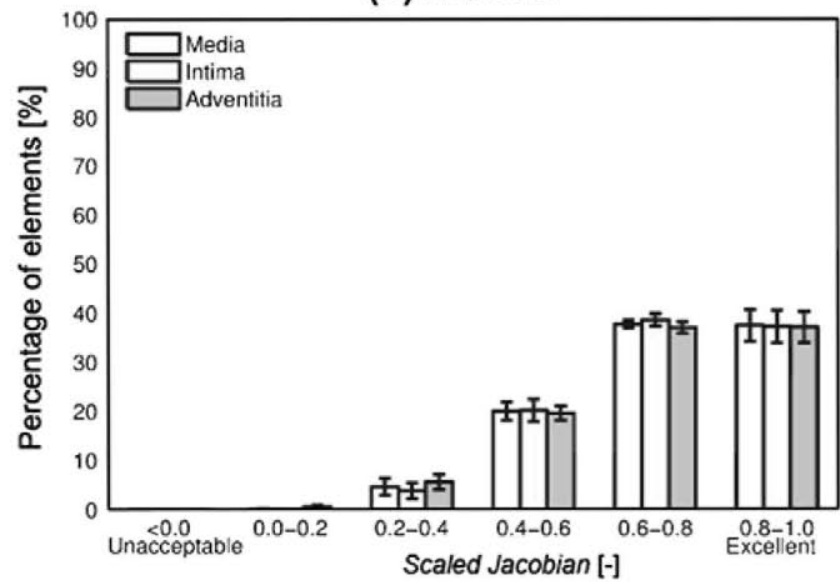

Fig. 7 Results for four representative abdominal aortic aneurysms (AAAs) and their corresponding intraluminal thrombi (ILTs) as distribution frequencies (\%) of the Scaled Jacobian values determined

In Figs. 6 and 7, recall that we remove elements with collapsed nodes from our mesh quality analysis as the calculation of the Scaled Jacobian degenerates in this special case of repeated nodes. Importantly, there are relatively few of these collapsed elements which are only used in the transition to the ILT. Their relative distribution in the ILT mesh is $16.0 \pm 4.03 \%(\mathrm{M} \pm \mathrm{SD})$, while their relative distribution in the entire mesh (aorta plus ILT) is $3.62 \pm 0.63 \%$. The application of collapsed elements in FE analysis may affect solution convergence and accuracy. Fortunately, at the transition to the ILT, we do not generally see large solution gradients, which are more difficult to capture. Nonetheless, stress distributions within collapsed elements should be viewed with caution. Elements with Scaled Jacobian values in the range of $0.5-1.0$ are well suited for FE analysis [2]. Negative values signify invalid (inverted) elements ill-suited for FE analysis. For the ILTs, the element quality is generally at a very high level: there are no elements with negative Scaled Jacobian values, and $94.8 \%$ of the total elements exhibit Scaled Jacobian values greater than 0.5. Importantly, note that the (layered) wall shapes are generally more complex than the thrombi shapes. Nonetheless, the elements of the AAA wall are also of high quality: $87.8 \%$ of elements in the wall exhibit Scaled Jacobian values greater than 0.5 , while only $0.22 \%$ fall below 0.2 . In the examples shown, we use approximately 25,000 elements per mesh (suitable for FE analysis), but the distribution of the Scaled Jacobian values does not change markedly with meshes of up to 125,000 elements (results not shown).

To study the distribution of relatively poor quality elements, we review a representative patient-specific AAA. To this end, Fig. 8 illustrates, for a single representative mesh, (b) ILT

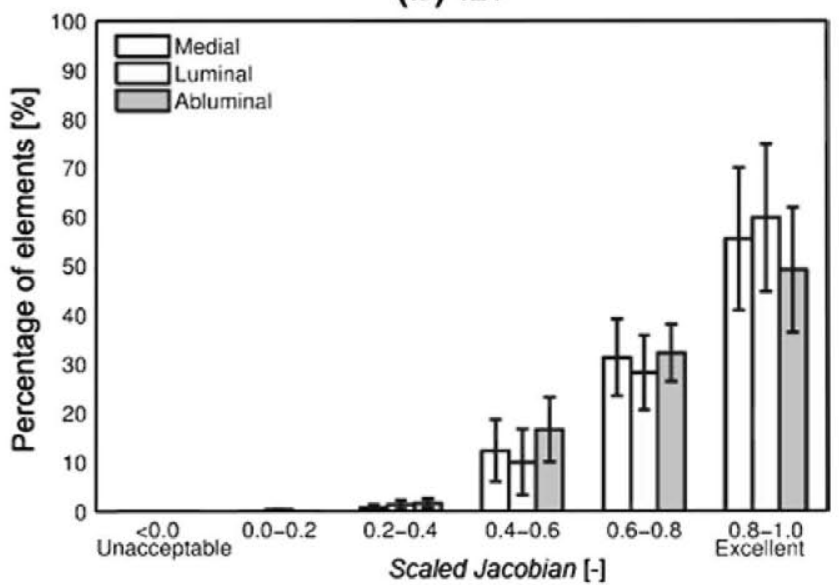

for individual layers: a the AAA wall and b the ILT. Bars represent the arithmetic means, and bracketed lines represent the standard errors (NB: we do not include collapsed elements in this analysis)

the Scaled Jacobian in the range of $0.0-0.5$, where we consider elements with Scaled Jacobian $>0.5$ as high-quality elements. The relatively low-quality elements do not occur in the region of the bifurcation, but rather at regions where the surfaces of the original geometry lie relatively parallel to the original MR imaging planes, either due to the presence of the ILT or due to the tortuosity inherent to the arteries.

\section{Discussion}

In this work, we propose a novel procedure for generating conforming hexahedral meshes of AAAs and their corresponding thrombi with high-quality elements, particularly in the area of the bifurcation into the iliac arteries. First, we separate the bifurcation into isolated vessels. We then divide each vessel into sections, each of which we mesh independently. Next, we build quadrilateral meshes of each isolated structure of the AAA. Finally, we generate conforming hexahedral meshes by adjusting and combining the quadrilateral meshes.

\subsection{Comparison to the state of the art}

In the literature, it is a relatively standard procedure to divide vessel bifurcations into independent vessels for meshing using a set of manually selected points. This procedure was first presented by Lee et al. [13], where they developed a method for meshing the lumen using hexahedral elements for studies in computational fluid dynamics. In this work, we use a modified approach to mesh not the lumen, but the AAA wall and corresponding ILT for solid 


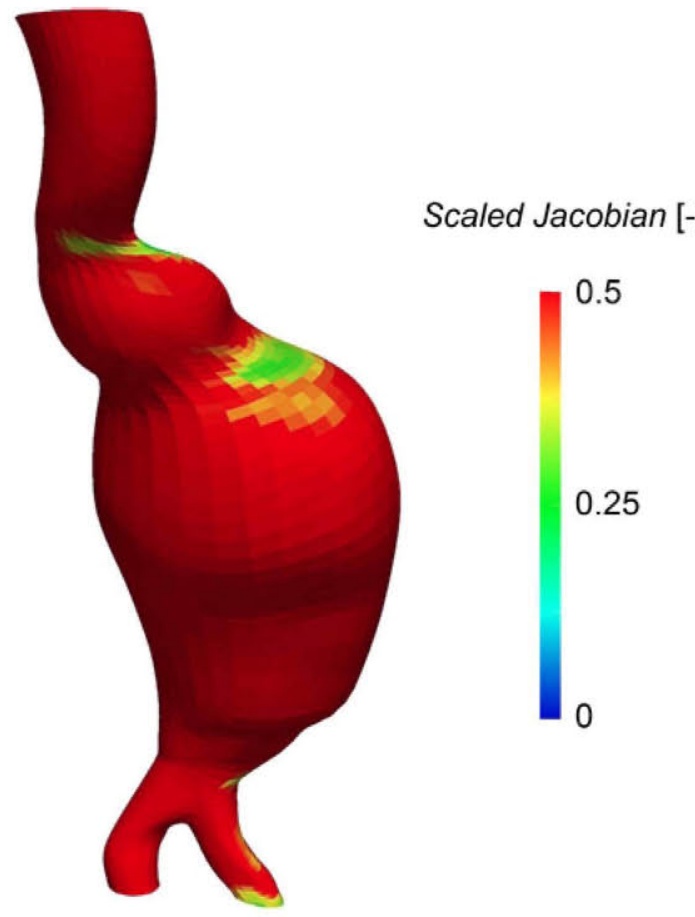

Fig. 8 Representative result for a patient-specific abdominal aortic aneurysm: Scaled Jacobian shown with range 0.0-0.5 to evaluate the lower quality elements. Relatively low-quality elements do not occur in the region of the bifurcation, but rather at regions where the surfaces of the original geometry lie relatively parallel to the original magnetic resonance imaging planes

analysis. In their method, the authors defined four points used to divide the bifurcation into separate vessels based on a $2 \mathrm{D}$ projection of the $3 \mathrm{D}$ model. In our application, the diseased state of the aorta results in the possibility of severe tortuosity in the iliac arteries. In such a case, the 2D projection proposed in the reported work breaks down, and hence, we define five points using the full 3D contours.

To put our method into context, we review the previous work in [6] who meshed a patient-specific AAA geometry determined from CTA using their custom method. Starting from the geometry of the lumen, the authors reconstructed the arterial wall by assuming a constant wall thickness and then mesh both the lumen and arterial wall using hexahedral elements. In their example, they do not account for the specific arterial layers (intima, media and adventitia) nor the thrombus or its layers. Our method affords more flexibility, and the division of the vessels (branching) into six independently modeled sections permits applying nonuniform distributions of elements. An inhomogeneous distribution is capable of generating smaller hexahedra in the regions of interest and larger hexahedra elsewhere. The proposed method permits defining the longitudinal and circumferential divisions of the vessels' sections, and a nonuniform distribution of the elements can be achieved as long as the number of the divisions is maintained. Currently, creating regions with a higher number of divisions (longitudinal or circumferential) than others is not considered, as it would suppose the use of collapsed hexahedra in the volume.

We use the Scaled Jacobian to evaluate the fitness of our hexahedral meshes for use in FE analysis. For a representative mesh, the majority of elements have Scaled Jacobian values greater than 0.5 , similar to the results presented in [6]. Both our method and that from [6] generate hexahedral meshes of the aortic wall (in our case also distinguishing between the arterial wall and thrombus, and including their respective layers) generated from geometries that evolve out of a single plane. While the results for mesh quality and computational time cannot be directly compared, we observe that for meshes of around 125,000 elements, both the distribution of Scaled Jacobian values and the percentage of elements inside the acceptable range $(0.5-1.0)$ are similar. However, De Santis et al. use a constant wall thickness for the artery, do not account for its layered morphology and do not have an ILT in their representative example. All of these details facilitate the likelihood of their method generating a higher portion of well-shaped elements. Furthermore, while our method appears to be significantly faster (131 vs. $1,892 \mathrm{~s}$ to generate a mesh of approximately 125,000 elements), the result depends on the specific examples meshed as well as the hardware and software implementation used to generate the result. It is important to note that we have not optimized our algorithmic implementation developed in MATLAB.

\subsection{Summary and future work}

Our method generates high-quality, patient-specific meshes of the AAA arterial wall and corresponding thrombus (including their layered morphologies) for use in FE analysis, e.g., in the evaluation of rupture risk of the aneurysm. Such improvements may provide greater fidelity in the $\mathrm{FE}$ estimates of, e.g., tissue stresses. We have tuned our MRI sequences to provide high contrast for the arterial tissue against the surrounding soft tissue, so that we determine the required information reliably. Our efficient method, based on manual segmentation of the MR Images that effectively distinguish between the different structures of interest, differentiates between the arterial wall and the ILT, and reconstructs a reasonable estimate of the actual wall thickness. Quality analysis on the four representative meshes shown here confirms that our method generates excellent meshes for use in FE analysis, even at the bifurcation regions where mesh generation is generally difficult.

Future work aims at improving the resulting mesh quality and further automation of the method. As a result of the ray-casting technique, relatively low-quality elements are 
likely generated in surface regions nearly parallel to the original MR Imaging slices. The application of sweeping techniques, which account for both the line tangent to the centerline and its normal plane, could improve the elements in these regions and thus improve the overall distribution of elements suitable for FE analysis. Additionally, an automated method for the selection of the vessel decomposition points could be implemented.

Acknowledgments The authors gratefully acknowledge the financial support of the European Commission under the 7th Framework Program, Grant Agreement Number 248782.

\section{References}

1. Antiga L, Ene-Iordache B, Caverni L, Cornalba GP, Remuzzi A (2002) Geometric reconstruction for computational mesh generation of arterial bifurcations from CT angiography. Comput Med Imaging Graph 26(4):227-235

2. Antiga L, Steinman DA (2004) Robust and objective decomposition and mapping of bifurcating vessels. IEEE Trans Med Imaging 23(6):704-713

3. Auricchio F, Conti M, De Beule M, De Santis G, Verhegghe B (2011) Carotid artery stenting simulation: from patient-specific images to finite element analysis. Med Eng Phys 33(3):281-289

4. De Santis G, Mortier P, De Beule M, Segers P, Verdonck P, Verhegghe B (2010) Patient-specific computational fluid dynamics: structured mesh generation from coronary angiography. Med Biol Eng Comput 48(4):371-380

5. De Santis G, De Beule M, Segers P, Verdonck P, Verhegghe B (2011) Patient-specific computational haemodynamics: generation of structured and conformal hexahedral meshes from triangulated surfaces of vascular bifurcations. Comput Methods Biomech Biomed Eng 14(9):797-802

6. De Santis G, De Beule M, Van Canneyt K, Segers P, Verdonck P, Verhegghe B (2011) Full-hexahedral structured meshing for image-based computational vascular modeling. Med Eng Phys 33(10):1318-1325

7. Baker TJ (1987) Three dimensional mesh generation by triangulation of arbitrary point sets. In: Proceedings of the 8th AIAA Computational fluid dynamics conference, Honolulu, HI, Paper $87-1124$

8. Gloviczki P, Ricotta JJWII (2007) Aneurysmal vascular disease. In: Townsend CM, Beauchamp RD, Evers BM, Mattox KL (eds) Sabiston textbook of surgery. Saunders Elsevier, Philadelphia

9. Henderson A (2007) The ParaView guide: a parallel visualization application. Kitware Inc., Clifton Park

10. Ho-Le K (1988) Finite element mesh generation methods: a review and classification. Comput Aided Des 20(1):27-38

11. Ito Y, Shih AM, Soni BK (2009) Octree-based reasonable-quality hexahedral mesh generation using a new set of refinement templates. Int J Numer Methods Eng 77(13):1809-1833

12. Knupp PM (2007) Remarks on mesh quality. In: Proceedings of the 45th AIAA aerospace sciences meeting and exhibit, Reno, NV

13. Lee S, Piersol N, Loth F, Fischer P, Leaf G, Smith B, Yedevalli R, Yardimci A, Alperin N, Schwartz L (2000) Automated mesh generation of an arterial bifurcation based upon in vivo MR images. In: Engineering in Medicine and Biology Society. Proceedings of the 22nd annual EMBS international conference, Chicago, IL, pp $719-722$

14. Löhner R, Parikh P (1988) Generation of three-dimensional unstructured grids by the advancing-front method. Int $J$ Numer Methods Fluids 8(10): 1135-1149

15. Longest PW, Vinchurkar S (2007) Effects of mesh style and grid convergence on particle deposition in bifurcating airway models with comparisons to experimental data. Med Eng Phys 29(3):350-366

16. Longo DL, Fauci AS, Kasper DL, Hauser SL, Jameson JL, Loscalzo J (2012) Harrison's manual of medicine. McGraw-Hill, New York

17. Lu JH-C, Song I, Quadros WR, Shimada K (2010) Pen-based user interface for geometric decomposition for hexahedral mesh generation. In: Proceedings of the 19th international meshing roundtable, Chattanooga, TN, pp 263-278

18. Mac Donald BJ (2007) Practical stress analysis with finite elements. Glasnevin Publishing, Dublin

19. Nichols M, Townsend N, Luengo-Fernandez R, Leal J, Gray A, Scarborough P, Rayner M (2012) European cardiovascular disease statistics 2012. European Heart Network, European Society of Cardiology, Brussels

20. Puso MA, Solberg J (2006) A stabilized nodally integrated tetrahedral. Int J Numer Methods Eng 67(6):841-867

21. Ramos A, Simões JA (2006) Tetrahedral versus hexahedral finite elements in numerical modeling of the proximal femur. Med Eng Phys 28(9):916-924

22. Schriefl AJ, Zeindlinger G, Pierce DM, Regitnig P, Holzapfel GA (2012) Determination of the layer-specific distributed collagen fibre orientations in human thoracic and abdominal aortas and common iliac arteries. J R Soc Interface 9(71):1275-1286

23. Shepherd JF, Johnson CR (2008) Hexahedral mesh generation constraints. Eng Comput 24(3):195-213

24. Stimpson CJ, Ernst CD, Knupp P, Pébay PP, Thompson D (2007) The Verdict geometric quality library. Sandia National Laboratories, SAND2007-1751

25. Tong J, Cohnert T, Regitnig P, Holzapfel GA (2011) Effects of age on the elastic properties of the intraluminal thrombus and the thrombus-covered wall in abdominal aortic aneurysms: biaxial extension behaviour and material modelling. Eur J Vasc Endovasc Surg 42(2):207-219

26. Venkatasubramaniam AK, Fagan MJ, Mehta T, Mylankal KJ, Ray B, Kuhan G, Chetter IC, McCollum PT (2004) A comparative study of aortic wall stress using finite element analysis for ruptured and non-ruptured abdominal aortic aneurysms. Eur J Vasc Endovasc Surg 28(2): 168-176

27. Verma CS, Fischer PF, Lee SE, Loth F (2005) An all-hex meshing strategy for bifurcation geometries in vascular flow simulation. In: Proceedings of 14 th international meshing roundtable, San Diego, CA, pp 363-375

28. Wassef M, Baxter BT, Chisholm RL, Dalman RL, Fillinger MF, Heinecke J, Humphrey JD, Kuivaniemi H, Parks WC, Pearce WH, Platsoucas CD, Sukhova GK, Thompson RW, Tilson MD, Zarins CK (2001) Pathogenesis of abdominal aortic aneurysms: a multidisciplinary research program supported by the National Heart, Lung, and Blood Institute. J Vasc Surg 34(4):730-738

29. Yerry MA, Shephard MS (1984) Automatic three-dimensional mesh generation by the modified-octree technique. Int J Numer Methods Eng 20(11):1965-1990

30. Zienkiewicz OC, Taylor RL, Zhu JZ (2005) The finite element method: its basis and fundamentals. Butterworth-Heinemann, Oxford 